Iustitia Socialis. Revista Arbitrada de Ciencias Jurídicas.

Año IV. Vol. IV. Nº 1. Edición Especial 2019

Hecho el depósito de Ley: FA2016000064 ISSN: 2542-3371

FUNDACIÓN KOINONIA (F.K). Santa Ana de Coro, Venezuela

Mallury Elizabeth Alcívar Tóala, Brenner Fabián Díaz Rodríguez

http://dx.doi.org/10.35381/racji.v4i1.545

\title{
Derechos reales: El derecho sobre el agua
}

Real rights: The right to water

\author{
Mallury Elizabeth Alcívar Tóala \\ mealcivar@sangregorio.edu.ec \\ Universidad San Gregorio de Portoviejo, Manabí \\ Ecuador. \\ https://orcid.org/0000-0001-7131-1330 \\ Brenner Fabián Díaz Rodríguez \\ bfdiaz@sangregorio.edu.ec \\ Universidad San Gregorio de Portoviejo, Manabí \\ Ecuador. \\ https://orcid.org/0000-0002-2872-9077
}

Recibido: 30 de octubre de 2019

Aprobado: 03 de diciembre de 2019

\section{RESUMEN}

El objetivo de este trabajo es fomentar el reconocimiento del acceso al agua potable, como derecho humano universal, indivisible e imprescriptible. La metodología utilizada fue documental, mediante la recopilación de información factible respecto al tema y análisis de la información donde se analizó, evaluó e interpretó las coincidencias y discordancias del material bibliográfico recopilado. Los principales resultados evidencian que todavía no existe un mercado de servicios ambientales, ni hidrológicos, las experiencias mencionadas operan en un rango de compensación indirecta hasta llegar a la compensación directa. Se concluye expresando que la dimensión cultural del agua es demasiado importante y política para ser ignorada o menospreciada.

Descriptores: Agua potable; abastecimiento de agua; tratamiento del agua; derechos humanos.

\section{ABSTRACT}

The objective of this work is to promote the recognition of access to drinking water, as a universal, indivisible and imprescriptible human right. The methodology used was documentary, through the collection of feasible information regarding the subject and analysis of the information where the coincidences and disagreements of the collected bibliographic material were analyzed, evaluated and interpreted. The main 
results show that there is still no market for environmental or hydrological services, the mentioned experiences operate in an indirect compensation range until direct compensation is reached. It is concluded by expressing that the cultural dimension of water is too important and political to be ignored or belittled.

Descriptors: Drinking water; water supply; water treatment; human rights.

\section{INTRODUCCIÓN}

El derecho real suele definirse como un señorío inmediato sobre un bien, y se refiere también a la relación directa entre el sujeto y la cosa, en contraposición con el derecho personal, consistente en una relación entre dos personas determinadas. Existen, al menos, dos criterios acerca de la distinción conceptual entre derechos reales y personales: el histórico y el filosófico - jurídico. En el derecho romano primitivo era imposible encontrar esta diferencia, ya que originalmente el derecho fue un poder, el del pater familias, que ejercía sobre todas las personas y cosas sometidas a su mancipium, sobre las cuales era soberano.

Posteriormente puede encontrarse la diferencia conceptual entre derechos personales y reales, cuando se sustituye el vínculo persona del deudor por la de sus bienes, pasándose de la ejecución personal a la ejecución patrimonial. ${ }^{1}$

Los derechos reales brotaron no sistemáticamente, sino empíricamente, de acuerdo con las necesidades socioeconómicas, y lo que los caracterizó fue que se les fue concediendo una actio in rem - vindicatio - con una progresiva extensión erga omnes, que en los comienzos sólo se concebía para el dominio o propiedad. ${ }^{2}$

Los primeros de esos derechos fueron las servidumbres rústicas, luego las urbanas. Después siguieron el usufructo y el uso, los derechos de habitatio y operae servorum, etc. Del derecho honorario se originará la enfiteusis, como así también las superficies. También de origen honorario, tomarán cuerpo los derechos reales de prenda e hipoteca, consideradas como de garantía.

Las expresiones ius in rem - ius in personam no aparecen en los textos romanos. Se

\footnotetext{
${ }^{1}$ Cabanellas, G., \& Cabanellas, G. (1979). Diccionario jurídico elemental. Heliasta.

2 Espinosa Lastra, M. B. (2015). La imposibilidad de cohabitar que conlleva el derecho de uso y habitación legal a favor de uno de los cónyuges cuando a la sociedad conyugal en disolución la conforma un único bien destinado a vivienda, vulnera el derecho constitucional a la vivienda del otro cónyuge (Bachelor's thesis, Loja)
} 
encuentran por primera vez en el Brachilogus iuris civilis o Corpus legum, redactado entre los siglos X y XII.

Fueron los glosadores y postglosadores quienes, sobre la base de que las acciones eran los medios procesales para tutelar los derechos concluyeron que los derechos protegidos por las actiones in rem y por las actiones in personam eran los ius in rem y los ius in personam, respectivamente. ${ }^{3}$ El artículo 583 del Código Civil ecuatoriano define las cosas o bienes como:

Art. 583.- Los bienes consisten en cosas corporales o incorporales. Corporales son las que tienen un ser real y pueden ser percibidas por los sentidos, como una casa, un libro. Incorporales las que consisten en meros derechos, como los créditos, y las servidumbres activas. ${ }^{4}$

Según Guillermo Allende: "Derecho Real es un derecho absoluto de contenido patrimonial cuyas normas de orden público establecen una relación entre persona y cosa, que previa publicidad obliga a la sociedad a abstenerse de actuar en contrario a ella, naciendo para el caso de violación una acción real que confiere a su titular la acción de preferencia y persecución de su derecho". Esta concepción es criticada por Marcel Planiol, quien manifiesta que no puede existir una relación entre persona y cosa, ya que las relaciones jurídicas solo se pueden dar entre personas, es decir siempre debe existir un sujeto activo y otro pasivo.

Pero Allende refuta esta crítica manifestando que es evidente que la relación debe ser entre personas y que dentro su concepto, en los Derechos Reales también existe esos sujetos, donde el sujeto activo es el titular de la cosa, quien puede realizar actos materiales y jurídicos sobre la cosa y el sujeto pasivo se constituiría en la sociedad que está obligada a respetar al titular en su dominio sobre la cosa. ${ }^{5}$

Luis Diez-Picazo define los derechos reales como Derechos Patrimoniales que consisten en un poder inmediato del titular sobre una cosa. "Derechos sobre las cosas". Manuel Albaladejo los considera el poder directo e inmediato sobre una cosa que concede a su titular un señorío, pleno (propiedad) o parcial (cosa ajena), sobre

\footnotetext{
${ }^{3}$ Bueres, A. J. (2012). Obligaciones "propter rem" y sus relaciones con otras figuras. Lecciones y Ensayos, 99127.

${ }^{4}$ Ecuatoriano, C. C. (2013). Código Civil Ecuatoriano. Codigo Civil Ecuatoriano

${ }^{5}$ Allesch Peñailillo, J., \& Obando Camino, I. (2005). El amparo del derecho de acceso a la información pública. lus et Praxis, 11(2), 103-155
} 
aquella, de forma que el ámbito de poder concedido tiene la cosa sometida a su dominación. En resumen se refieren a la relación jurídica que surge entre las personas respecto de los bienes. ${ }^{6}$

Art. 595.- Derecho real es el que tenemos sobre una cosa sin respecto a determinada persona. Son derechos reales el de dominio, el de herencia, los de usufructo, uso o habitación, los de servidumbres activas, el de prenda y el de hipoteca. De estos derechos nacen las acciones reales. $^{7}$

En algunos casos el nombre se liga al derecho objetivo, al que da las reglas, y entonces se habla del Derecho de cosas (Código Civil alemán, Código Civil portugués), aludiéndose así al conjunto de normas que en el respectivo cuerpo legal gobierna o regula los derechos subjetivos que se tienen directamente sobre las cosas. En otros casos, el título no considera el derecho objetivo sino que alude a los derechos subjetivos, y entonces se habla De los derechos reales (Código Civil argentino, Código Civil suizo).

En el Código Civil de Etiopía de 1960, el libro sobre los derechos reales lleva el amplio título De los bienes. Es el mismo que en sus obras adopta la mayoría de los autores franceses y chilenos. Se toma aquí la palabra en dos acepciones: en la de cosas sobre las que recaen los derechos reales y en la de los derechos reales mismos. Pues, de acuerdo con un sentir, que se precia de lógico y no sofisticado, los derechos son igualmente bienes, cosas incorporales, y aún podría decirse que son los verdaderos bienes, ya que las cosas por sí solas valen menos que los derechos que sobre ellas puedan tenerse, por lo cual .terminan estos pensadores, hablando con propiedad, son los derechos los que representan un valor, un bien y no las cosas mismas.

Consideración especial merece el Código Civil italiano de 1942. Su Libro III se titula simplemente "De la propiedad", aun cuando se ocupa también de todos los demás derechos reales. ¿Por qué esa denominación? Seguramente porque ninguno de los otros derechos reales deja de estar ligado al dominio o propiedad, que es el derecho

\footnotetext{
${ }^{6}$ Blanco, L. E. A., \& MENÉNDEZ, M. D. C. D. (2012). La desnaturalización de la finalidad del registro de propiedad inmueble en el Perú (Doctoral dissertation, Pontificia Universidad Católica del Perú. Facultad de Derecho).

${ }^{7}$ Ecuatoriano, C. C. (2013). Código Civil Ecuatoriano. Codigo Civil Ecuatoriano
} 
real por excelencia.

El derecho real es el poder directo e inmediato sobre una cosa, poder o señorío que, dentro de los márgenes de la ley, puede ser más amplio o menos amplio. La mayor o menor amplitud depende del contenido del derecho de que se trate. Por ejemplo, el dominio o propiedad es el derecho más amplio, porque contiene el cúmulo máximo de facultades; en contraste, el derecho real de habitación es uno de los más limitados, pues se reduce sólo a la facultad de morar en una casa. ${ }^{8}$

Se considera bien todo aquello, material o inmaterial, que de manera objetiva, tiene una valoración económica (o valor en el mercado), así como relevancia jurídica, es jurídicamente susceptible de apropiación privada y cuyo fin es ser objeto de una relación jurídica:

Art. 583.- Los bienes consisten en cosas corporales o incorporales. Corporales son las que tienen un ser real y pueden ser percibidas por los sentidos, como una casa, un libro. Incorporales las que consisten en meros derechos, como los créditos, y las servidumbres activas. ${ }^{9}$

El derecho real es absoluto, por derecho absoluto se entiende aquel derecho cuyo titular puede hacerlo valer contra todos los demás sujetos (erga omnes), porque se estima que sobre todos ellos pesa el deber de no perturbar ni violar el derecho mismo. El número de derechos reales es cerrado (numerus clausus), es decir, sólo existen los tipos que las leyes expresamente establecen. Los derechos reales se distinguen, según se ha explicado antes, por su contenido. En cuanto a relaciones de señorío autónomo sobre la cosa, pueden contener dos categorías de facultades:

a) la facultad de disfrute directo de la cosa (en relación a la cual tiene ésta un valor de uso y es un bien directo), o sea, el goce de los frutos (frui) y el uso directo de la cosa (uti);

b) la facultad de utilización indirecta de la misma, o sea, de su valor de cambio; aquí la cosa es sólo un bien instrumental, un medio para obtener dinero. ${ }^{10}$

Así, pues, los derechos reales se dividen en:

a) derechos reales de goce o disfrute, entre los cuales se encuentran el derecho

\footnotetext{
${ }^{8}$ Vodanovic, A. (2003). Manual de derecho civil. Ed. Jur. ConoSur.

${ }^{9}$ Ecuatoriano, C. C. (2013). Código Civil Ecuatoriano. Codigo Civil Ecuatoriano

${ }^{10}$ De las Casas, G. B. (2016). RESEÑA HISTÓRICA SOBRE LA CATEGORÍA DE LOS DERECHOS REALES Y LA OPONIBILIDAD ERGA OMNES.
} 
de propiedad (el más amplio señorío de goce sobre una cosa) y otros derechos con un poder más limitado de goce o disfrute.

b) los derechos reales de goce limitado, a saber: usufructo, uso, habitación, servidumbre

c) derechos reales de garantía; su contenido es el poder de provocar (en la forma procesal adecuada) la venta forzosa de la cosa para obtener dinero; son derechos reales limitados de garantía: la prenda y la hipoteca

En el Derecho Romano dominó el sistema del numerus clausus; no había más derechos reales que el dominio, las servidumbres, la enfiteusis, la superficie, el usufructo, el uso, la habitación y los derechos de garantía, prenda e hipoteca. Por el contrario, en las legislaciones germánicas se admitía que los particulares dieran efecto real a cualquiera obligación referida a una cosa determinada. ${ }^{11}$

Se consideran derechos reales:

- Propiedad

- Posesión

- Usufructo.

- Uso y Habitación

- Superficie

- Servidumbre

Derechos reales de garantía:

- Prenda

- Anticresis

- Hipoteca

- Retención

Casos especiales no Reales:

- Arrendamiento

- Comodato

- Embargo.

Art. 715.- Posesión es la tenencia de una cosa determinada con ánimo de señor o dueño; sea que el dueño o el que se da por tal tenga la cosa por sí mismo, o

\footnotetext{
${ }^{11}$ Alterini, E. G. J. H. (1992). El derecho real. Editorial Abeledo-Perrot SAE el Buenos Aires, Argentina.
} 
bien por otra persona en su lugar y a su nombre. El poseedor es reputado dueño, mientras otra persona no justifica serlo.

Art. 716.- Se puede poseer una cosa por varios títulos.

Art. 717.- La posesión puede ser regular o irregular. Se llama posesión regular la que procede de justo título y ha sido adquirida de buena fe, aunque la buena fe no subsista después de adquirida la posesión. Se puede ser, por consiguiente, poseedor regular y poseedor de mala fe, como viceversa el poseedor de buena fe puede ser poseedor irregular. Si el título es translativo de dominio, es también necesaria la tradición. La posesión de una cosa a ciencia y paciencia del que se obligó a entregarla, hará presumir la tradición; a menos que ésta haya debido efectuarse por la inscripción del título.

Art. 718.- El justo título es constitutivo o translativo de dominio. Son constitutivos de dominio la ocupación, la accesión y la prescripción. Son translaticios de dominio los que, por su naturaleza, sirven para transferirlo como la venta, la permuta, la donación entre vivos. Pertenecen a esta clase las sentencias de adjudicación en juicios divisorios, y los actos legales de partición. Las sentencias judiciales sobre derechos litigiosos no forman nuevo título para legitimar la posesión. Las transacciones, en cuanto se limitan a reconocer o declarar derechos preexistentes, no forman nuevo título; pero, en cuanto transfieren la propiedad de un objeto no disputado, constituyen un título nuevo. ${ }^{12}$

En el derecho real interviene un solo sujeto que es el titular de la potestad sobre la cosa. Implica un poder sobre una cosa y es un derecho absoluto, oponible erga omnesy su configuración del contenido de la ley. Precisan para constituirse del título y el modo (acto ostensible de transmisión de la posesión), y son susceptibles de ser adquiridos por usucapión.

El derecho real concede a favor de su titular dos derechos accesorios; El de persecución, es decir el titular del derecho real tiene la facultad de perseguir la cosa cuando ella se encuentra en manos de terceros, buscando su reivindicación. Y el de preferencia, porque se tiene la facultad de ser pagado con preferencia a otros acreedores, en virtud de la calidad real de su crédito.

\footnotetext{
${ }^{12}$ Ecuatoriano, C. C. (2013). Código Civil Ecuatoriano. Codigo Civil Ecuatoriano
} 
Los derechos reales son oponibles a todo el mundo, porque toda la sociedad está obligada a respetar el derecho propietario de cada persona y, según la doctrina, los derechos Reales se clasifican en Principales y accesorios, donde las principales son la propiedad, el usufructo, el uso y las servidumbres y los Derechos Reales Accesorios serian la hipoteca y la prenda. ${ }^{13}$

Art. 599.- El dominio, que se llama también propiedad, es el derecho real en una cosa corporal, para gozar y disponer de ella, conforme a las disposiciones de las leyes y respetando el derecho ajeno, sea individual o social. La propiedad separada del goce de la cosa, se llama mera o nuda propiedad. ${ }^{14}$

Las facultades materiales del dueño son:

Derecho de poseer: el dominio es un derecho real que se ejerce por la posesión; tiene defensas posesorias. Así como también posee acción reivindicatoria.

Derecho de usar: puede servirse de la cosa como le plazca.

Derecho de gozar: puede percibir todos los frutos que la cosa produce por su sólo título.

Derecho de disponer materialmente: puede alterar la materialidad de la cosa, cambiar su aspecto, destruirla, etcétera. El ejercicio de esta facultad es posible siempre que no se atente contra la propiedad de un tercero. ${ }^{15}$

En cuanto a las facultades jurídicas, tiene:

Derecho de enajenar.

Derecho de gravar.

Art. 602.- Las cosas que la naturaleza ha hecho comunes a todos los hombres, como la alta mar, no son susceptibles de dominio, y ninguna nación, corporación o individuo tiene derecho de apropiárselas. Su uso y goce se determinan, entre individuos de una nación, por las leyes de ésta; y entre distintas naciones, por el Derecho Internacional. Art. 603.Los modos de adquirir el dominio son la ocupación, la accesión, la tradición, la sucesión por causa de muerte y la prescripción ${ }^{16}$

\footnotetext{
${ }^{13}$ Alterini, E. G. J. H. (1992). El derecho real. Editorial Abeledo-Perrot SAE el Buenos Aires, Argentina.

${ }^{14}$ Ecuatoriano, C. C. (2013). Código Civil Ecuatoriano. Codigo Civil Ecuatoriano

${ }^{15}$ Lathrop Gómez, F. (2011). Procedencia de la acción meramente declarativa del dominio en el derecho chileno. Ius et Praxis, 17(2), 3-24.

${ }^{16}$ Ecuatoriano, C. C. (2013). Código Civil Ecuatoriano. Codigo Civil Ecuatoriano
} 


\section{Derecho de constituir derechos personales.}

\section{Derecho de abandonar.}

Art. 612.- Los ríos y todas las aguas que corren por cauces naturales, así como los lagos naturales, son bienes nacionales de uso público. También son bienes nacionales de uso público las vertientes que nacen y mueren dentro de una misma heredad. No hay ni se reconoce derechos de dominio adquiridos sobre ellas y los preexistentes solo se limitan a su uso en cuanto sea eficiente y de acuerdo con la Ley de Aguas. En cuanto a la extensión del dominio de las riberas de dichos ríos, aguas y lagos, se estará a lo que dispongan las leyes especiales. Para los efectos determinados en el segundo inciso de este artículo, se entenderá que mueren en una heredad, no solo las aguas que no salen de la misma, sino aquellas que, dentro de la heredad, desembocan en otra corriente de agua, a la cual quedan incorpora

Se han presentado una serie de documentos legales que permiten introducir sobre los derechos de propiedad en concordancia con los espacios donde se encuentra el agua, la cual es un derecho humano universal el acceso a la misma, por lo que intereses particulares no pueden prevalecer sobre el derecho colectivo.

\section{MÉTODO}

Se abordó una investigación documental - jurídica con la finalidad de analizar el tema del agua como derecho fundamental para la humanidad, teniéndose un diseño bibliográfico que permitió la selección, organización, interpretación y análisis de información referida al tema, por lo que la población de estudio se basó en documentación jurídica inherente al tema, aplicándose la técnica de análisis de contenido para su comprensión, teniéndose como fundamento metodológico la postura de Palella Stracuzzi y Martins Pestana (2012).

\section{RESULTADOS}

Teniendo en cuenta que el agua es considerada un bien público ¿se considera lícito el pago por su consumo?

El pago por servicios ambientales se ha visto como una alternativa para darle "valor económico" a los bosques o ecosistemas naturales que luego de retirar la madera han sido tradicionalmente considerados de bajo valor y por ende justificar su protección o conservación. En Quito, Ecuador, la Fundación Antisana con el apoyo 
de "Nature Conservancy" y la Agencia de los Estados Unidos para el Desarrollo Internacional (USAID) elaboraron la idea de un nuevo fondo de agua- FONAG dedicado a financiar la protección de las cuencas alrededor de la ciudad. FONAG es un fondo de fideicomiso de dotación no decreciente que puede recibir dinero del gobierno y de organizaciones particulares y ONG.

Los recursos son manejados por una entidad financiera independiente que invierte los fondos y los réditos de las inversiones se emplean para la protección de las cuencas. No se agota el fondo de dotación. La lógica de usar solamente los réditos financieros es asegurar la disponibilidad de los recursos en el futuro.

Otra experiencia interesante con la participación de la empresa municipal de servicios públicos es la de ETAPA, en la ciudad de Cuenca en el Ecuador. Siendo una empresa líder a nivel nacional, ha desarrollado un programa de gestión ambiental integral que comenzó con la adquisición de tierras en las cuencas que proveen el agua potable de la ciudad. A medida que se consolidó el programa de compra y protección de tierras, la empresa amplió sus frentes de acción hacía la descontaminación de las aguas.

Es así que la empresa desarrolla un programa para recolectar los aceites usados, una causa importante de contaminación del agua, como también construyó la primera planta de tratamiento de aguas servidas en el país. Actualmente, la empresa ha contabilizado la cantidad de recursos que requiere para proteger sus fuentes estimativo de cinco centavos por metro cubico de agua potable tratada por la empresa. ${ }^{17}$

La heterogeneidad de experiencias expuestas demuestra que todavía no existe un mercado de servicios ambientales, ni hidrológicos, como tal. Las experiencias mencionadas operan en un rango de compensación indirecta hasta llegar a la compensación directa. Aunque podríamos decir que es un mercado mixto en la medida que se está retribuyendo monetariamente por un servicio, consideramos que

\footnotetext{
${ }^{17}$ Echavarria, M., La Pinta, C., La Rábida, E. A. L., \& Quito, E. C. U. A. D. O. R. (2003, June). Algunas lecciones sobre la aplicación de pagos por la protección del agua con base en experiencias en Colombia y Ecuador. In III Congreso Latinoamericano de Manejo de Cuencas Hidrográficas (Arequipa, Perú, 9 al 13 de junio de 2003).
} 
es importante resaltar que hay información imperfecta entre los participantes del mercado. No se conoce exactamente lo que se está vendiendo y comprando.

Otro punto importante es que el contexto legal en el que opera un mercado es fundamental y usualmente los economistas tienden a ignorarlo. El cambio de uso de la tierra está regulado y el agua es un bien de dominio público. Por lo tanto, el pago por los servicios hidrológicos debe de ser coherente con el régimen forestal y del agua para no debilitar la autoridad del Estado. También para prevenir que el mecanismo de pago se convierta en una forma de coima por buen comportamiento o extorsión de parte de los dueños de tierras. El pago es por la protección de los servicios hidrológicos, en vez de la provisión del agua.

\section{CONCLUSIONES}

En este sentido, es vital resaltar que los pagos por servicios ambientales son mecanismos que no pueden ser aplicados a cualquier situación y momento. Este tipo de mecanismo económico tiene particularidades que requieren ciertas condiciones para aplicarse exitosamente. Uno de estos requisitos es la tenencia de la tierra que para aplicar mecanismos de pagos por la protección de los servicios ambientales deben de estar definidos si no se puede estar generando incentivos perversos a la deforestación. En otras palabras, el conflicto de tierras puede agudizarse al existir una posibilidad de ingresos para aquellos que argumenten ser dueños de las tierras y por ende se precipite la expansión de la frontera agrícola.

La dimensión cultural del agua es demasiado importante y política para ser ignorada o menospreciada. Para las comunidades rurales y en particular las indígenas, el agua es parte de la vida y la relación con la Madre Tierra (Declaratoria de Kyoto, 2003). Esto ha llevado a una posición internacional que defiende el agua como un derecho humano y como tal se contrapone con cualquier iniciativa para introducirla dentro de una dinámica de mercado.

Vogel (2002) presenta una propuesta que puede ir dilucidando esta controversia: el agua debe ser visto como un derecho y un bien. El acceso al agua suficiente para cubrir las necesidades básicas debe ser un derecho fundamental del ciudadano. $Y$ en ese sentido, el agua debe proveerse a toda la población y gratis o a muy bajo 
Iustitia Socialis. Revista Arbitrada de Ciencias Jurídicas.

Año IV. Vol. IV. Nº 1. Edición Especial 2019

Hecho el depósito de Ley: FA2016000064 ISSN: 2542-3371

FUNDACIÓN KOINONIA (F.K). Santa Ana de Coro, Venezuela

Mallury Elizabeth Alcívar Tóala, Brenner Fabián Díaz Rodríguez

costo para las comunidades de escasos recursos. Sin embargo, el agua que se consume más allá de la subsistencia, pasa a ser un bien económico que debe ser pagado en forma progresiva; mientras más se usa más se paga. El precio debe señalar los límites naturales de mantener y regenerar la calidad y el flujo - aspecto usualmente ignorado. ${ }^{18}$

\section{REFERENCIAS CONSULTADAS}

1. Allesch Peñailillo, J., \& Obando Camino, I. (2005). El amparo del derecho de acceso a la información pública. lus et Praxis, 11(2), 103-155

2. Alterini, E. G. J. H. (1992). El derecho real. Editorial Abeledo-Perrot SAE el Buenos Aires, Argentina.

3. Blanco, L. \& menéndez, M. (2012). La desnaturalización de la finalidad del registro de propiedad inmueble en el Perú (Doctoral dissertation, Pontificia Universidad Católica del Perú. Facultad de Derecho).

4. Bueres, A. J. (2012). Obligaciones "propter rem" y sus relaciones con otras figuras. Lecciones y Ensayos, 99-127.

5. Cabanellas, G., \& Cabanellas, G. (1979). Diccionario jurídico elemental. Heliasta.

6. De las Casas, G. (2016). Reseña histórica sobre la categoría de los derechos reales y la oponibilidad erga omnes.

7. Ecuatoriano, C. (2013). Código Civil Ecuatoriano. Código Civil Ecuatoriano.

8. Echavarría, M., La Pinta, C., La Rábida, E. A. L., \& Quito, E. C. U. A. D. O. R. (2003). Algunas lecciones sobre la aplicación de pagos por la protección del agua con base en experiencias en Colombia y Ecuador. In III Congreso Latinoamericano de Manejo de Cuencas Hidrográficas (Arequipa, Perú, 9 al 13 de junio de 2003).

9. Espinosa Lastra, M. B. (2015). La imposibilidad de cohabitar que conlleva el derecho de uso y habitación legal a favor de uno de los cónyuges cuando a la sociedad conyugal en disolución la conforma un único bien destinado a

\footnotetext{
${ }^{18}$ Echavarria, M., La Pinta, C., La Rábida, E. A. L., \& Quito, E. C. U. A. D. O. R. (2003, June). Algunas lecciones sobre la aplicación de pagos por la protección del agua con base en experiencias en Colombia y Ecuador. In III Congreso Latinoamericano de Manejo de Cuencas Hidrográficas (Arequipa, Perú, 9 al 13 de junio de 2003).
} 
vivienda, vulnera el derecho constitucional a la vivienda del otro cónyuge (Bachelor's thesis, Loja).

10. Lathrop Gómez, F. (2011). Procedencia de la acción meramente declarativa del dominio en el derecho chileno. lus et Praxis, 17(2), 3-24.

11. Vodanovic, A. (2003). Manual de derecho civil. Ed. Jur. ConoSur.

\section{REFERENCES CONSULTED}

1. Allesch Peñailillo, J., \& Obando Camino, I. (2005). The protection of the right of access to public information. lus et Praxis, 11 (2), 103-15

2. Alterini, E. G. J. H. (1992). The real right Editorial Abeledo-Perrot SAE and Buenos Aires, Argentina.

3. Blanco, L. \& menéndez, M. (2012). The denaturalization of the purpose of real estate registration in Peru (Doctoral dissertation, Pontifical Catholic University of Peru. Law School).

4. Bueres, A. J. (2012). Obligations "propter rem" and its relations with other figures. Lessons and Essays, 99-127.

5. Cabanellas, G., \& Cabanellas, G. (1979). Elementary legal dictionary. Heliasta

6. De las Casas, G. (2016). Historical review of the category of real rights and enforceability erga omnes.

7. Ecuatoriano, C. (2013). Ecuadorian Civil Code. Ecuadorian Civil Code.

8. Echavarría, M., La Pinta, C., La Rábida, E. A. L., \& Quito, E. C. U. A. D. O. R. (2003). Some lessons on the application of payments for water protection based on experiences in Colombia and Ecuador. In III Latin American Congress of River Basin Management (Arequipa, Peru, June 9-13, 2003).

9. Espinosa Lastra, M. B. (2015). The impossibility of cohabiting that entails the right of use and legal room in favor of one of the spouses when the dissolving conjugal society is formed by a single property intended for housing, violates the constitutional right to housing of the other spouse (Bachelor's thesis, Loja)

10. Lathrop Gómez, F. (2011). Origin of the merely declarative action of the domain in Chilean law. Ius et Praxis, 17 (2), 3-24.

11. Vodanovic, A. (2003). Manual of civil law. Ed. Jur. ConoSur. 
Iustitia Socialis. Revista Arbitrada de Ciencias Jurídicas.

Año IV. Vol. IV. N 1. Edición Especial 2019

Hecho el depósito de Ley: FA2016000064 ISSN: 2542-3371

FUNDACIÓN KOINONIA (F.K). Santa Ana de Coro, Venezuela

Mallury Elizabeth Alcívar Tóala, Brenner Fabián Díaz Rodríguez

C2019 por los autores. Este artículo es de acceso abierto y distribuido según los términos y condiciones de la licencia Creative Commons Atribución-NoComercial-Compartirlgual 4.0 Internacional (CC BY-NC-SA 4.0) (https://creativecommons.org/licenses/by-nc-sa/4.0/). 